\title{
Fourier Stability Analysis of Two Finite Element Schemes for Reaction-Diffusion System with Fast Reversible Reaction
}

\author{
Ann Jalal \\ annsawoor@yahoo.com \\ Mohammed O. Al-Amr \\ malamroo@yahoo.com \\ Department of Mathematics, \\ College of Computer Sciences and Mathematics, \\ University of Mosul, Mosul, Iraq
}

Received on: 09/10/2012

Accepted on: 04/12/2012

\begin{abstract}
In this paper, the stability analysis is performed on two Galerkin finite element schemes for solving reaction-diffusion system with fast reversible reaction. Fourier (Von Neumann) method is implemented to propose time-step criteria for the consistent and the lumped schemes with four popular choices for $\theta$. We have found that the two schemes are unconditionally stable when $1 / 2 \leq \theta \leq 1$, while the consistent scheme is stable under the conditions $\Delta t \leq(\Delta x)^{2} / 6 a$ and $\Delta t \leq(\Delta x)^{2} / 6 b$ when $\theta=0$. Also, the lumped scheme is stable under the conditions $\Delta t \leq(\Delta x)^{2} / 2 a$ and $\Delta t \leq(\Delta x)^{2} / 2 b$ when $\theta=0$.
\end{abstract}

Keywords: finite element scheme, reaction-diffusion system, consistent scheme, lumped scheme

$$
\begin{aligned}
& \text { تحليل استقرارية فوريير لطريقتي العناصر المحددة لنظام التفاعل-الانتشار مع تفاعل عكسي سريع }
\end{aligned}
$$

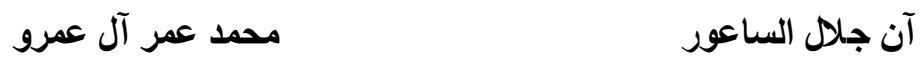

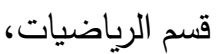

$$
\begin{aligned}
& \text { كلية علوم الحاسوب والرياضيات، } \\
& \text { جامعة الموصل، الموصل، العراق }
\end{aligned}
$$

$$
\begin{aligned}
& \text { الملخص } \\
& \text { في هذا البحث، تم تحليل الاستقرارية لطريقتي العناصر المحدة لكليركن لحل نظام التقاعل-الانتشار مع } \\
& \text { تفاعل عكسي سريع. ولقد تم تطبيق طريقة فوريير (فون نيومان) لايجاد معيار خطوة الزمن للطريقتين المتسقة }
\end{aligned}
$$

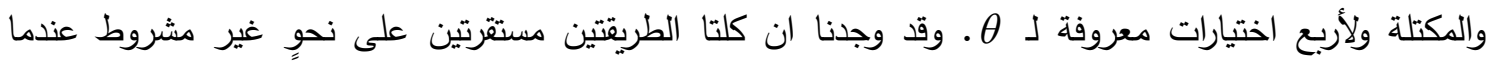

$$
\begin{aligned}
& \text { } \frac{1}{2} \leq \theta \leq 1 \\
& \text { o=0 } 0 \\
& \text {. } \theta=0
\end{aligned}
$$


الكلمات المفتاحية: طريقة العناصر المحددة، نظام التفاعل-الانتثار، الطريقة المتسقة، الطريقة المكتلة.

\section{Introduction.}

Stability is a condition on the numerical solution, namely that all errors, such as round-off errors (due to the finite arithmetic of the computer) must remain bounded when the iteration process advances. That is, for finite values of $\Delta t$ and $\Delta x$, the error has to remain bounded, when the number of time steps tends to infinity [6].

Nevertheless, stability is probably the most pressing problem in any algorithm, since it is necessary rather than sufficient condition for accuracy. It is generally the first one to be encountered in any attempt to obtain a solution. Accuracy and computational efficiency follow after one is assured that a meaningful computation can be carried out successfully. Moreover, experience indicates that an unstable scheme is not convergent [8].

Many methods have been developed for the analysis of stability; nearly, all of them restricted to linear problems. But, even with this restriction, the investigation of stability for initial, boundary value problems can be extremely complicated, particularly in the presence of boundary conditions and their numerical representation [6].

At present, several techniques are available to analyze linear stability. This includes the discrete perturbation method, the Hirt method, the matrix method and the Von Neumann method. Comparing with other techniques, the Von Neumann method is the most widely applied technique [11].

In recent years, many authors have successfully applied the Fourier (Von Neumann) method to analyze the stability of finite element schemes [1-3,7].

The present paper has been organized as follows: Section 2 deals with the methodology of the Fourier (Von Neumann) method. In Sections 3 and 4, we analyze linear stability of the consistent and the lumped finite element schemes, respectively, for the reaction-diffusion system by using the Fourier (Von Neumann) method. Practical application of this analysis is provided in Section 5. Conclusions are given in Section 6.

In this work, we consider the reversible chemical reaction of the type $2 A \square B$, the reaction-diffusion system will take the form [4]:

$u_{t}=a u_{x x}-2 k\left(k_{1} u^{2}-k_{2} v\right)$, in $\Omega \times(0, T)$,

$v_{t}=b v_{x x}+k\left(k_{1} u^{2}-k_{2} v\right), \quad$ in $\Omega \times(0, T)$,

with the boundary conditions

$\frac{\partial u}{\partial x}=\frac{\partial v}{\partial x}=0, \quad$ on $\partial \Omega \times(0, T)$,

and the initial conditions

$u(x, 0)=u_{0}(x), v(x, 0)=v_{0}(x)$, in $\Omega$,

where $u(x, t)$ and $v(x, t)$ are the concentrations of $A$ and $B$ respectively, $a, b>0$ are diffusion coefficients, $k_{1}, k_{2}$ are rate constants for the reactions $2 A \rightarrow B$ and $B \rightarrow 2 A$ respectively, $k$ is the chemical kinetics factor.

\section{Methodology of Fourier (Von Neumann) Method}

The method developed by John Von Neumann has an interesting history. It started in Los Alamos during the World War II as he was part of the team of high-level 
scientists developing the first nuclear device. Consequently, the method was classified as 'secret', until its brief description in Crank and Nicholson (1947) and in a publication by Charney et al. (1950) [6].

The methodology for the application of the Von Neumann stability condition can be summarized as follows $[6,10]$ :

1. Replace, in the numerical scheme, all the terms of the form $u_{n+q}^{m+p}$ by $\zeta^{m+p} e^{i \beta(n+q) \Delta x}$ where $\zeta=e^{\gamma \Delta t}$ represent the Amplification Factor or Amplitude, $\beta>0$ is the wave number of $x, \gamma>0$ is the wave number of $t, p$ is determined according to the number of time levels, $q$ is determined according to the number of space levels and $i=\sqrt{-1}$.

2. As all the terms contain the factor $\zeta^{m} e^{i \beta n \Delta x}$, the next step is to simplify all the terms by this factor

3. From the obtained relation, derive the explicit form of the amplification factor $\zeta$.

4. Check the Von Neumann stability condition, i.e., $|\zeta| \leq 1$, which will provide an easy way for distinguishing between unstable, conditionally stable or unconditionally stable schemes.

\section{Stability Analysis of the Consistent Finite Element Scheme}

This method supposes that the solutions can be separated by the forms:

$u_{n+q}^{m+p}=\zeta_{1}^{m+p} e^{i \beta(n+q) \Delta x}$,

$v_{n+q}^{m+p}=\zeta_{2}^{m+p} e^{i \beta(n+q) \Delta x}$,

where $p$ will take the values $p=0$ and $p=1$ for two time level scheme and $q$ will take the values $q=-1, q=0$ and $q=1$ for three space level scheme.

To apply this method, the system (1) must be in the linearized form [5,10]

$$
\begin{aligned}
& \frac{\partial u}{\partial t}=a \frac{\partial^{2} u}{\partial x^{2}}+2 k k_{2} v, \\
& \frac{\partial v}{\partial t}=b \frac{\partial^{2} v}{\partial x^{2}}-k k_{2} v,
\end{aligned}
$$

For some values of $k$ and $k_{2}$, we can neglect the terms which contain $k k_{2}$, so we have

$\frac{\partial u}{\partial t}=a \frac{\partial^{2} u}{\partial x^{2}}$,

$\frac{\partial v}{\partial t}=b \frac{\partial^{2} v}{\partial x^{2}}$

For the equations (5.a) and (5.b), assembling the equations of the elements $x_{n-1} \leq x \leq x_{n}$ and $x_{n} \leq x \leq x_{n+1}$, we write the recurrence relations at node $x_{n}$ as:

$\left(-\frac{\theta a}{\Delta x}+\frac{\Delta x}{6 \Delta t}\right) u_{n-1}^{m+1}+\left(\frac{2 \theta a}{\Delta x}+\frac{2 \Delta x}{3 \Delta t}\right) u_{n}^{m+1}+\left(-\frac{\theta a}{\Delta x}+\frac{\Delta x}{6 \Delta t}\right) u_{n+1}^{m+1}$
$=\left(\frac{(1-\theta) a}{\Delta x}+\frac{\Delta x}{6 \Delta t}\right) u_{n-1}^{m}+\left(-\frac{2(1-\theta) a}{\Delta x}+\frac{2 \Delta x}{3 \Delta t}\right) u_{n}^{m}+\left(\frac{(1-\theta) a}{\Delta x}+\frac{\Delta x}{6 \Delta t}\right) u_{n+1}^{m}$,

and

$\left(-\frac{\theta b}{\Delta x}+\frac{\Delta x}{6 \Delta t}\right) v_{n-1}^{m+1}+\left(\frac{2 \theta b}{\Delta x}+\frac{2 \Delta x}{3 \Delta t}\right) v_{n}^{m+1}+\left(-\frac{\theta b}{\Delta x}+\frac{\Delta x}{6 \Delta t}\right) v_{n+1}^{m+1}$ 


$$
=\left(\frac{(1-\theta) b}{\Delta x}+\frac{\Delta x}{6 \Delta t}\right) v_{n-1}^{m}+\left(-\frac{2(1-\theta) b}{\Delta x}+\frac{2 \Delta x}{3 \Delta t}\right) v_{n}^{m}+\left(\frac{(1-\theta) b}{\Delta x}+\frac{\Delta x}{6 \Delta t}\right) v_{n+1}^{m} .
$$

To apply Fourier (Von Neumann) method [6], we substitute $u_{n+q}^{m+p}=\zeta_{1}^{m+p} e^{i \beta(n+q) \Delta x}$, $v_{n+q}^{m+p}=\zeta_{2}^{m+p} e^{i \beta(n+q) \Delta x}$ in the equations (6) and (7) respectively, we obtain $\left(-\frac{\theta a}{\Delta x}+\frac{\Delta x}{6 \Delta t}\right) \zeta_{1}^{m+1} e^{i \beta(n-1) \Delta x}+\left(\frac{2 \theta a}{\Delta x}+\frac{2 \Delta x}{3 \Delta t}\right) \zeta_{1}^{m+1} e^{i \beta n \Delta x}+\left(-\frac{\theta a}{\Delta x}+\frac{\Delta x}{6 \Delta t}\right) \zeta_{1}^{m+1} e^{i \beta(n+1) \Delta x}$ $=\left(\frac{(1-\theta) a}{\Delta x}+\frac{\Delta x}{6 \Delta t}\right) \zeta_{1}^{m} e^{i \beta(n-1) \Delta x}+\left(-\frac{2(1-\theta) a}{\Delta x}+\frac{2 \Delta x}{3 \Delta t}\right) \zeta_{1}^{m} e^{i \beta n \Delta x}+\left(\frac{(1-\theta) a}{\Delta x}+\frac{\Delta x}{6 \Delta t}\right) \zeta_{1}^{m} e^{i \beta(n+1) \Delta x}$, and

$$
\begin{aligned}
& \left(-\frac{\theta b}{\Delta x}+\frac{\Delta x}{6 \Delta t}\right) \zeta_{2}^{m+1} e^{i \beta(n-1) \Delta x}+\left(\frac{2 \theta b}{\Delta x}+\frac{2 \Delta x}{3 \Delta t}\right) \zeta_{2}^{m+1} e^{i \beta n \Delta x}+\left(-\frac{\theta b}{\Delta x}+\frac{\Delta x}{6 \Delta t}\right) \zeta_{2}^{m+1} e^{i \beta(n+1) \Delta x} \\
& =\left(\frac{(1-\theta) b}{\Delta x}+\frac{\Delta x}{6 \Delta t}\right) \zeta_{2}^{m} e^{i \beta(n-1) \Delta x}+\left(-\frac{2(1-\theta) b}{\Delta x}+\frac{2 \Delta x}{3 \Delta t}\right) \zeta_{2}^{m} e^{i \beta n \Delta x}+\left(\frac{(1-\theta) b}{\Delta x}+\frac{\Delta x}{6 \Delta t}\right) \zeta_{2}^{m} e^{i \beta(n+1) \Delta x} .
\end{aligned}
$$

Dividing the above equations by $\zeta_{1}^{m} e^{i \beta n \Delta x}$ and $\zeta_{2}^{m} e^{i \beta n \Delta x}$ respectively and simplifying, to obtain

$$
\begin{aligned}
& \left(\left(-\frac{\theta a}{\Delta x}+\frac{\Delta x}{6 \Delta t}\right)\left(e^{i \beta \Delta x}+e^{-i \beta \Delta x}\right)+\frac{2 \theta a}{\Delta x}+\frac{2 \Delta x}{3 \Delta t}\right) \zeta_{1}=\left(\frac{(1-\theta) a}{\Delta x}+\frac{\Delta x}{6 \Delta t}\right)\left(e^{i \beta \Delta x}+e^{-i \beta \Delta x}\right) \\
& -\frac{2(1-\theta) a}{\Delta x}+\frac{2 \Delta x}{3 \Delta t},
\end{aligned}
$$

and

$$
\begin{aligned}
& \left(\left(-\frac{\theta b}{\Delta x}+\frac{\Delta x}{6 \Delta t}\right)\left(e^{i \beta \Delta x}+e^{-i \beta \Delta x}\right)+\frac{2 \theta b}{\Delta x}+\frac{2 \Delta x}{3 \Delta t}\right) \zeta_{2}=\left(\frac{(1-\theta) b}{\Delta x}+\frac{\Delta x}{6 \Delta t}\right)\left(e^{i \beta \Delta x}+e^{-i \beta \Delta x}\right) \\
& -\frac{2(1-\theta) b}{\Delta x}+\frac{2 \Delta x}{3 \Delta t} .
\end{aligned}
$$

Using the relation $e^{i \beta \Delta x}+e^{-i \beta \Delta x}=2 \cos (\beta \Delta x)$ and simplifying, we derive the explicit form of the amplification factors as follows:

$$
\zeta_{1}=\frac{-\frac{4(1-\theta) a}{\Delta x} \sin ^{2}\left(\frac{\beta \Delta x}{2}\right)+\frac{\Delta x}{3 \Delta t}\left(3-2 \sin ^{2}\left(\frac{\beta \Delta x}{2}\right)\right)}{\frac{4 \theta a}{\Delta x} \sin ^{2}\left(\frac{\beta \Delta x}{2}\right)+\frac{\Delta x}{3 \Delta t}\left(3-2 \sin ^{2}\left(\frac{\beta \Delta x}{2}\right)\right)},
$$

and

$$
\zeta_{2}=\frac{-\frac{4(1-\theta) b}{\Delta x} \sin ^{2}\left(\frac{\beta \Delta x}{2}\right)+\frac{\Delta x}{3 \Delta t}\left(3-2 \sin ^{2}\left(\frac{\beta \Delta x}{2}\right)\right)}{\frac{4 \theta b}{\Delta x} \sin ^{2}\left(\frac{\beta \Delta x}{2}\right)+\frac{\Delta x}{3 \Delta t}\left(3-2 \sin ^{2}\left(\frac{\beta \Delta x}{2}\right)\right)} .
$$

To satisfy the Von Neumann stability condition, we must have $\left|\zeta_{1}\right| \leq 1,\left|\zeta_{2}\right| \leq 1$, i.e.,

$$
-1 \leq \frac{-\frac{4(1-\theta) a}{\Delta x} \sin ^{2}\left(\frac{\beta \Delta x}{2}\right)+\frac{\Delta x}{3 \Delta t}\left(3-2 \sin ^{2}\left(\frac{\beta \Delta x}{2}\right)\right)}{\frac{4 \theta a}{\Delta x} \sin ^{2}\left(\frac{\beta \Delta x}{2}\right)+\frac{\Delta x}{3 \Delta t}\left(3-2 \sin ^{2}\left(\frac{\beta \Delta x}{2}\right)\right)} \leq 1,
$$

and 


$$
-1 \leq \frac{-\frac{4(1-\theta) b}{\Delta x} \sin ^{2}\left(\frac{\beta \Delta x}{2}\right)+\frac{\Delta x}{3 \Delta t}\left(3-2 \sin ^{2}\left(\frac{\beta \Delta x}{2}\right)\right)}{\frac{4 \theta b}{\Delta x} \sin ^{2}\left(\frac{\beta \Delta x}{2}\right)+\frac{\Delta x}{3 \Delta t}\left(3-2 \sin ^{2}\left(\frac{\beta \Delta x}{2}\right)\right)} \leq 1 .
$$

Since $0 \leq \sin ^{2}(\beta \Delta x / 2) \leq 1$ and $0 \leq \theta \leq 1$ so that, the right-hand sides of the above inequalities are always true.

To satisfy the left-hand sides of the above inequalities, this depends on the value of $\theta$ which has the following choices [9]:

1. Forward Difference Method: when $\theta=0$, we obtain

$$
\frac{4 a}{\Delta x} \sin ^{2}\left(\frac{\beta \Delta x}{2}\right) \leq \frac{2 \Delta x}{3 \Delta t}\left(3-2 \sin ^{2}\left(\frac{\beta \Delta x}{2}\right)\right) \text {, }
$$

and

$$
\frac{4 b}{\Delta x} \sin ^{2}\left(\frac{\beta \Delta x}{2}\right) \leq \frac{2 \Delta x}{3 \Delta t}\left(3-2 \sin ^{2}\left(\frac{\beta \Delta x}{2}\right)\right) .
$$

The worst situation occurs when $\sin ^{2}(\beta \Delta x / 2)=1$, so that, the scheme is conditionally stable with the following conditions:

$$
\Delta t \leq \frac{(\Delta x)^{2}}{6 a},
$$

and

$$
\Delta t \leq \frac{(\Delta x)^{2}}{6 b} .
$$

2. Central Difference Method: when $\theta=1 / 2$, we obtain

$$
0 \leq \frac{2 \Delta x}{3 \Delta t}\left(3-2 \sin ^{2}\left(\frac{\beta \Delta x}{2}\right)\right)
$$

and

$$
0 \leq \frac{2 \Delta x}{3 \Delta t}\left(3-2 \sin ^{2}\left(\frac{\beta \Delta x}{2}\right)\right) .
$$

So that, the scheme is unconditionally stable.

3. Galerkin's Method: when $\theta=2 / 3$, we obtain

$$
-\frac{4 a}{3 \Delta x} \sin ^{2}\left(\frac{\beta \Delta x}{2}\right) \leq \frac{2 \Delta x}{3 \Delta t}\left(3-2 \sin ^{2}\left(\frac{\beta \Delta x}{2}\right)\right),
$$

and

$$
-\frac{4 b}{3 \Delta x} \sin ^{2}\left(\frac{\beta \Delta x}{2}\right) \leq \frac{2 \Delta x}{3 \Delta t}\left(3-2 \sin ^{2}\left(\frac{\beta \Delta x}{2}\right)\right) .
$$

Hence, the scheme is unconditionally stable.

4. Backward Difference Method: when $\theta=1$, we obtain

$$
-\frac{4 a}{\Delta x} \sin ^{2}\left(\frac{\beta \Delta x}{2}\right) \leq \frac{2 \Delta x}{3 \Delta t}\left(3-2 \sin ^{2}\left(\frac{\beta \Delta x}{2}\right)\right),
$$

and

$$
-\frac{4 b}{\Delta x} \sin ^{2}\left(\frac{\beta \Delta x}{2}\right) \leq \frac{2 \Delta x}{3 \Delta t}\left(3-2 \sin ^{2}\left(\frac{\beta \Delta x}{2}\right)\right) .
$$


So that, the scheme is unconditionally stable.

\section{Stability Analysis of the Lumped Finite Element Scheme}

For the equations (5.a) and (5.b), assembling the equations of the elements $x_{n-1} \leq x \leq x_{n}$ and $x_{n} \leq x \leq x_{n+1}$, we write the recurrence relations at node $x_{n}$ as:

$$
\begin{aligned}
& -\frac{\theta a}{\Delta x} u_{n-1}^{m+1}+\left(\frac{2 \theta a}{\Delta x}+\frac{\Delta x}{\Delta t}\right) u_{n}^{m+1}-\frac{\theta a}{\Delta x} u_{n+1}^{m+1} \\
& =\frac{(1-\theta) a}{\Delta x} u_{n-1}^{m}+\left(-\frac{2(1-\theta) a}{\Delta x}+\frac{\Delta x}{\Delta t}\right) u_{n}^{m}+\frac{(1-\theta) a}{\Delta x} u_{n+1}^{m},
\end{aligned}
$$

and

$$
\begin{aligned}
& -\frac{\theta b}{\Delta x} v_{n-1}^{m+1}+\left(\frac{2 \theta b}{\Delta x}+\frac{\Delta x}{\Delta t}\right) v_{n}^{m+1}-\frac{\theta b}{\Delta x} v_{n+1}^{m+1} \\
& =\frac{(1-\theta) b}{\Delta x} v_{n-1}^{m}+\left(-\frac{2(1-\theta) b}{\Delta x}+\frac{\Delta x}{\Delta t}\right) v_{n}^{m}+\frac{(1-\theta) b}{\Delta x} v_{n+1}^{m} .
\end{aligned}
$$

To apply Fourier (Von Neumann) method, we substitute $u_{n+q}^{m+p}=\zeta_{1}^{m+p} e^{i \beta(n+q) \Delta x}$, $v_{n+q}^{m+p}=\zeta_{2}^{m+p} e^{i \beta(n+q) \Delta x}$ in the equations (8) and (9) respectively, we obtain

$$
\begin{aligned}
& -\frac{\theta a}{\Delta x} \zeta_{1}^{m+1} e^{i \beta(n-1) \Delta x}+\left(\frac{2 \theta a}{\Delta x}+\frac{\Delta x}{\Delta t}\right) \zeta_{1}^{m+1} e^{i \beta n \Delta x}-\frac{\theta a}{\Delta x} \zeta_{1}^{m+1} e^{i \beta(n+1) \Delta x} \\
& =\frac{(1-\theta) a}{\Delta x} \zeta_{1}^{m} e^{i \beta(n-1) \Delta x}+\left(-\frac{2(1-\theta) a}{\Delta x}+\frac{\Delta x}{\Delta t}\right) \zeta_{1}^{m} e^{i \beta n \Delta x}+\frac{(1-\theta) a}{\Delta x} \zeta_{1}^{m} e^{i \beta(n+1) \Delta x},
\end{aligned}
$$

and

$$
\begin{aligned}
& -\frac{\theta b}{\Delta x} \zeta_{2}^{m+1} e^{i \beta(n-1) \Delta x}+\left(\frac{2 \theta b}{\Delta x}+\frac{\Delta x}{\Delta t}\right) \zeta_{2}^{m+1} e^{i \beta n \Delta x}-\frac{\theta b}{\Delta x} \zeta_{2}^{m+1} e^{i \beta(n+1) \Delta x} \\
& =\frac{(1-\theta) b}{\Delta x} \zeta_{2}^{m} e^{i \beta(n-1) \Delta x}+\left(-\frac{2(1-\theta) b}{\Delta x}+\frac{\Delta x}{\Delta t}\right) \zeta_{2}^{m} e^{i \beta n \Delta x}+\frac{(1-\theta) b}{\Delta x} \zeta_{2}^{m} e^{i \beta(n+1) \Delta x} .
\end{aligned}
$$

Dividing the above equations by $\zeta_{1}^{m} e^{i \beta n \Delta x}$ and $\zeta_{2}^{m} e^{i \beta n \Delta x}$ respectively and simplifying, to obtain

$\left(-\frac{\theta a}{\Delta x}\left(e^{i \beta \Delta x}+e^{-i \beta \Delta x}\right)+\frac{2 \theta a}{\Delta x}+\frac{\Delta x}{\Delta t}\right) \zeta_{1}=\frac{(1-\theta) a}{\Delta x}\left(e^{i \beta \Delta x}+e^{-i \beta \Delta x}\right)-\frac{2(1-\theta) a}{\Delta x}+\frac{\Delta x}{\Delta t}$,

and

$$
\left(-\frac{\theta b}{\Delta x}\left(e^{i \beta \Delta x}+e^{-i \beta \Delta x}\right)+\frac{2 \theta b}{\Delta x}+\frac{\Delta x}{\Delta t}\right) \zeta_{2}=\frac{(1-\theta) b}{\Delta x}\left(e^{i \beta \Delta x}+e^{-i \beta \Delta x}\right)-\frac{2(1-\theta) b}{\Delta x}+\frac{\Delta x}{\Delta t} .
$$

Using the relation $e^{i \beta \Delta x}+e^{-i \beta \Delta x}=2 \cos (\beta \Delta x)$ and simplifying, we derive the explicit form of the amplification factors as follows:

$$
\zeta_{1}=\frac{-\frac{4(1-\theta) a}{\Delta x} \sin ^{2}\left(\frac{\beta \Delta x}{2}\right)+\frac{\Delta x}{\Delta t}}{\frac{4 \theta a}{\Delta x} \sin ^{2}\left(\frac{\beta \Delta x}{2}\right)+\frac{\Delta x}{\Delta t}},
$$

and 


$$
\zeta_{2}=\frac{-\frac{4(1-\theta) b}{\Delta x} \sin ^{2}\left(\frac{\beta \Delta x}{2}\right)+\frac{\Delta x}{\Delta t}}{\frac{4 \theta b}{\Delta x} \sin ^{2}\left(\frac{\beta \Delta x}{2}\right)+\frac{\Delta x}{\Delta t}} .
$$

To satisfy the Von Neumann stability condition, we must have $\left|\zeta_{1}\right| \leq 1,\left|\zeta_{2}\right| \leq 1$, i.e.,

$$
-1 \leq \frac{-\frac{4(1-\theta) a}{\Delta x} \sin ^{2}\left(\frac{\beta \Delta x}{2}\right)+\frac{\Delta x}{\Delta t}}{\frac{4 \theta a}{\Delta x} \sin ^{2}\left(\frac{\beta \Delta x}{2}\right)+\frac{\Delta x}{\Delta t}} \leq 1,
$$

and

$$
-1 \leq \frac{-\frac{4(1-\theta) b}{\Delta x} \sin ^{2}\left(\frac{\beta \Delta x}{2}\right)+\frac{\Delta x}{\Delta t}}{\frac{4 \theta b}{\Delta x} \sin ^{2}\left(\frac{\beta \Delta x}{2}\right)+\frac{\Delta x}{\Delta t}} \leq 1 .
$$

Since, $0 \leq \sin ^{2}(\beta \Delta x / 2) \leq 1$ and $0 \leq \theta \leq 1$ so that, the right-hand sides of the above inequalities are always true.

To satisfy the left-hand sides of the above inequalities, this depends on the value of $\theta$ which has the following choices [9]:

1. Forward Difference Method: when $\theta=0$, we obtain

$\frac{4 a}{\Delta x} \sin ^{2}\left(\frac{\beta \Delta x}{2}\right) \leq \frac{2 \Delta x}{\Delta t}$,

and

$\frac{4 b}{\Delta x} \sin ^{2}\left(\frac{\beta \Delta x}{2}\right) \leq \frac{2 \Delta x}{\Delta t}$.

The worst situation occurs when $\sin ^{2}(\beta \Delta x / 2)=1$, so that, the scheme is conditionally stable with the following conditions:

$$
\Delta t \leq \frac{(\Delta x)^{2}}{2 a}
$$

and

$$
\Delta t \leq \frac{(\Delta x)^{2}}{2 b} .
$$

2. Central Difference Method: when $\theta=1 / 2$, we obtain

$$
\begin{aligned}
& 0 \leq \frac{2 \Delta x}{\Delta t}, \\
& \text { and } \\
& 0 \leq \frac{2 \Delta x}{\Delta t} .
\end{aligned}
$$

So that, the scheme is unconditionally stable.

3. Galerkin's Method: when $\theta=2 / 3$, we obtain

$-\frac{4 a}{3 \Delta x} \sin ^{2}\left(\frac{\beta \Delta x}{2}\right) \leq \frac{2 \Delta x}{\Delta t}$,

and 


$$
-\frac{4 b}{3 \Delta x} \sin ^{2}\left(\frac{\beta \Delta x}{2}\right) \leq \frac{2 \Delta x}{\Delta t} .
$$

Hence, the scheme is unconditionally stable.

4. Backward Difference Method: when $\theta=1$, we obtain

$-\frac{4 a}{\Delta x} \sin ^{2}\left(\frac{\beta \Delta x}{2}\right) \leq \frac{2 \Delta x}{\Delta t}$,

and

$$
-\frac{4 b}{\Delta x} \sin ^{2}\left(\frac{\beta \Delta x}{2}\right) \leq \frac{2 \Delta x}{\Delta t} \text {. }
$$

So that, the scheme is unconditionally stable.

\section{Practical Application}

We consider the reaction of the reversible dimerization of $o$-phenylenedioxydimethylsilane (2, 2-dimethyl-1, 2, 3-benzodioxasilole) which has been described by the following reaction-diffusion system [4]:

$u_{t}=a u_{x x}-2 k\left(k_{1} u^{2}-k_{2} v\right)$, in $[0,0.1] \times(0, T)$,

$v_{t}=b v_{x x}+k\left(k_{1} u^{2}-k_{2} v\right), \quad$ in $[0,0.1] \times(0, T)$,

with the boundary conditions

$u_{x}(0, t)=u_{x}(0.1, t)=v_{x}(0, t)=v_{x}(0.1, t)=0$,

and the initial conditions $u_{0}$ and $v_{0}$ are defined as follows:

$u_{0}(x)= \begin{cases}0 & \text { for } x \in[0,0.03] \\ \frac{1}{2} \sin \left(\frac{50 \pi}{7}(x-0.03)\right) & \text { for } x \in[0.03,0.1],\end{cases}$

and

$v_{0}(x)= \begin{cases}\frac{1}{4} \cos \left(\frac{50 \pi}{7} x\right) & \text { for } x \in[0,0.07] \\ 0 & \text { for } x \in[0.07,0.1],\end{cases}$

The rate constants for both reactions can be estimated at the temperature $T=298 \mathrm{~K}$ which as follows:

$$
k_{1} \approx 1.072 \cdot 10^{-4} \mathrm{~L}^{2} \mathrm{~mol}^{-2} \text { and } k_{2} \approx 2.363 \cdot 10^{-6} \mathrm{~L}^{2} \mathrm{~mol}^{-2},
$$

and diffusion coefficients

$$
a \approx 1.579 \cdot 10^{-9} \mathrm{~m}^{2} \mathrm{~s}^{-1} \text { and } b \approx 1.042 \cdot 10^{-9} \mathrm{~m}^{2} \mathrm{~s}^{-1} .
$$

It is possible to choose the chemical kinetics factor where $k>0$.

To check the validity of the obtained stability properties for the consistent scheme, we plot the amplification factors $\zeta_{1}$ and $\zeta_{2}$ for all the wavenumbers in the range $\varphi \in(-\pi, \pi)$, where $\varphi=\beta \Delta x[1]$, i.e.,

$$
\zeta_{1}=\frac{-\frac{4(1-\theta) a}{\Delta x} \sin ^{2}\left(\frac{\varphi}{2}\right)+\frac{\Delta x}{3 \Delta t}\left(3-2 \sin ^{2}\left(\frac{\varphi}{2}\right)\right)}{\frac{4 \theta a}{\Delta x} \sin ^{2}\left(\frac{\varphi}{2}\right)+\frac{\Delta x}{3 \Delta t}\left(3-2 \sin ^{2}\left(\frac{\varphi}{2}\right)\right)},
$$


and

$$
\zeta_{2}=\frac{-\frac{4(1-\theta) b}{\Delta x} \sin ^{2}\left(\frac{\varphi}{2}\right)+\frac{\Delta x}{3 \Delta t}\left(3-2 \sin ^{2}\left(\frac{\varphi}{2}\right)\right)}{\frac{4 \theta b}{\Delta x} \sin ^{2}\left(\frac{\varphi}{2}\right)+\frac{\Delta x}{3 \Delta t}\left(3-2 \sin ^{2}\left(\frac{\varphi}{2}\right)\right)} .
$$

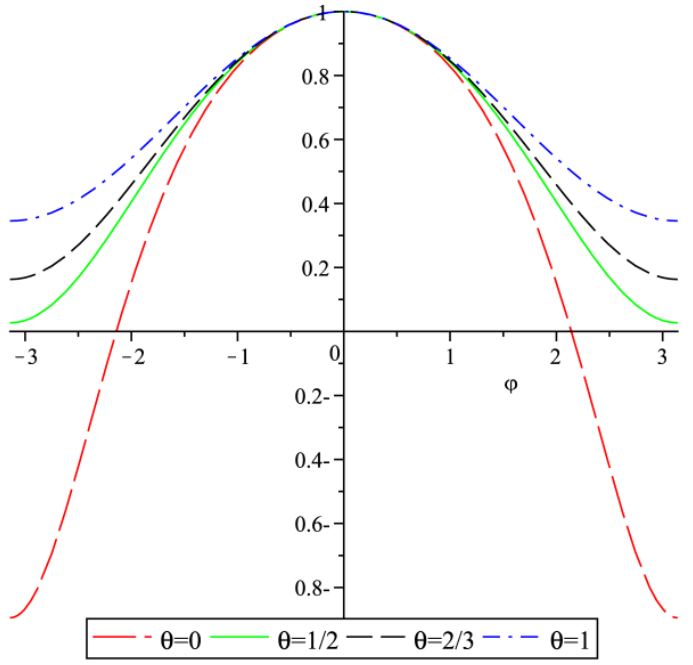

(a)

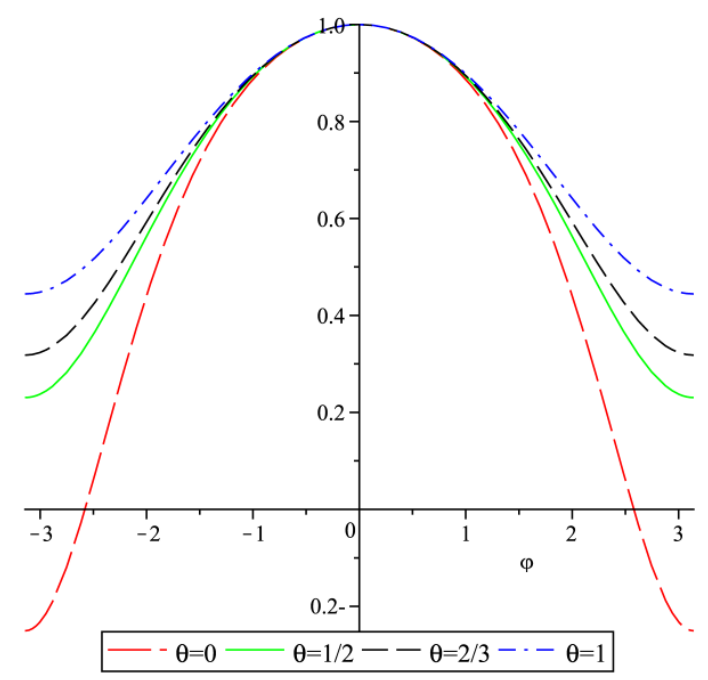

(b)

Fig.1. (a) the amplification factor $\zeta_{1}$ (b) the amplification factor $\zeta_{2}$ with wavenumber $\varphi \in(-\pi, \pi)$ for the consistent scheme with different choices for $\theta$ when $\Delta x=0.001$ and

$$
\Delta t=100
$$

The amplification factors $\zeta_{1}$ and $\zeta_{2}$ do not exceed 1.0 for any wavenumber for any choice of $\theta$ because the central, Galerkin and backward, consistent schemes are unconditionally stable, while the stability conditions of the forward, consistent scheme are satisfied (see Fig.1).

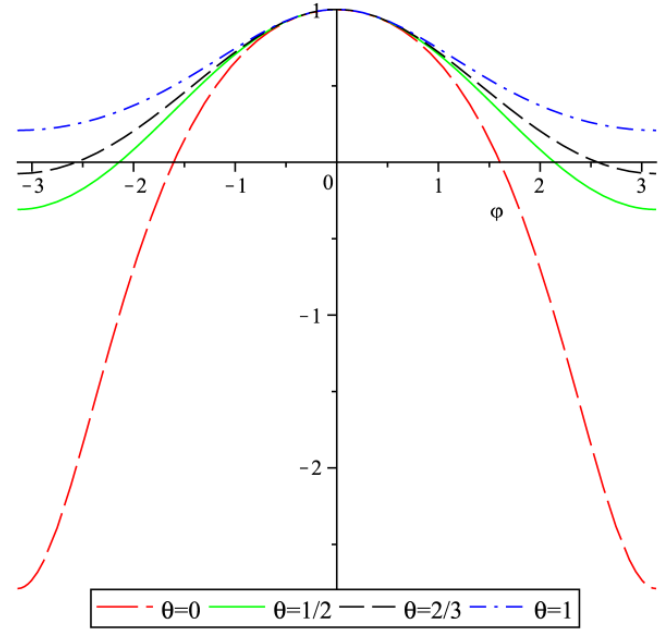

(a)

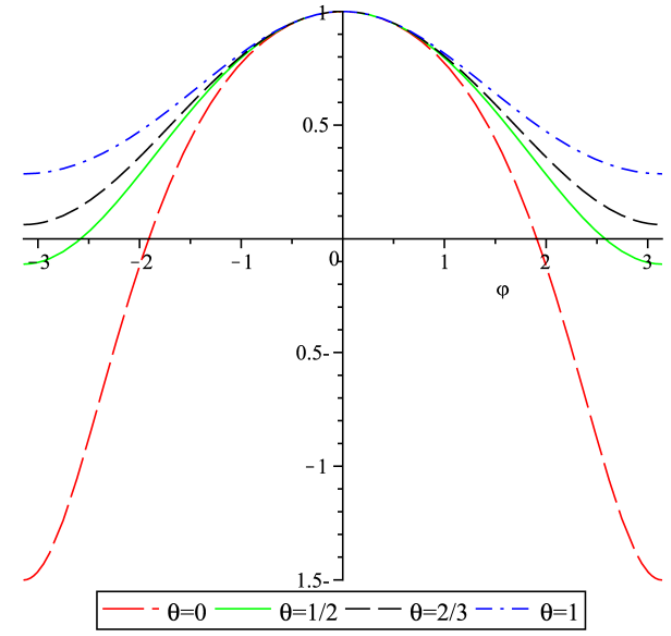

(b) 
Fig.2. (a) the amplification factor $\zeta_{1}(b)$ the amplification factor $\zeta_{2}$ with wavenumber $\varphi \in(-\pi, \pi)$ for the consistent scheme with different choices for $\theta$ when $\Delta x=0.001$ and

$$
\Delta t=200
$$

The amplification factors $\zeta_{1}$ and $\zeta_{2}$ for the forward, consistent scheme increase to exceed 1.0 with the increase of $\Delta t$, i.e. $\zeta_{1}$ exceed 1.0 when $\Delta t>105.5520372$ and $\zeta_{2}$ exceed 1.0 when $\Delta t>159.9488164$, because the stability conditions are not satisfied (see Fig.2).

To check the validity of the obtained stability properties for the lumped scheme, we plot the amplification factors $\zeta_{1}$ and $\zeta_{2}$ for all the wavenumbers in the range $\varphi \in(-\pi, \pi)$, where $\varphi=\beta \Delta x$,i.e.,

$$
\zeta_{1}=\frac{-\frac{4(1-\theta) a}{\Delta x} \sin ^{2}\left(\frac{\varphi}{2}\right)+\frac{\Delta x}{\Delta t}}{\frac{4 \theta a}{\Delta x} \sin ^{2}\left(\frac{\varphi}{2}\right)+\frac{\Delta x}{\Delta t}},
$$

and

$$
\zeta_{2}=\frac{-\frac{4(1-\theta) b}{\Delta x} \sin ^{2}\left(\frac{\varphi}{2}\right)+\frac{\Delta x}{\Delta t}}{\frac{4 \theta b}{\Delta x} \sin ^{2}\left(\frac{\varphi}{2}\right)+\frac{\Delta x}{\Delta t}} .
$$

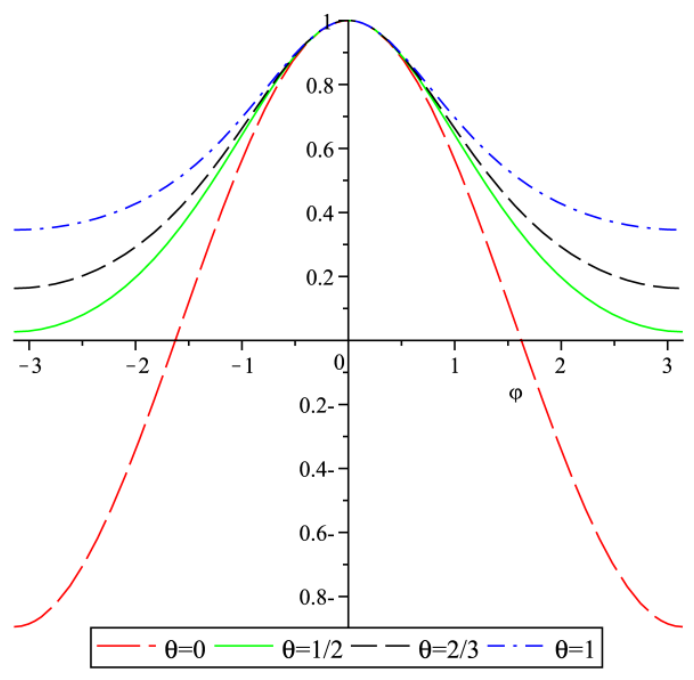

(a)

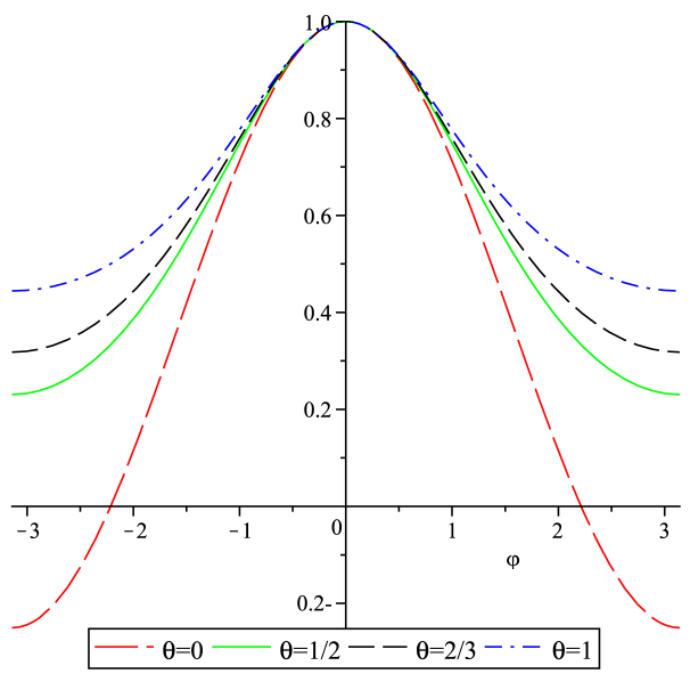

(b)

Fig.3. (a) the amplification factor $\zeta_{1}$ (b) the amplification factor $\zeta_{2}$ with wavenumber $\varphi \in(-\pi, \pi)$ for the lumped scheme with different choices for $\theta$ when $\Delta x=0.001$ and

$$
\Delta t=300
$$

The amplification factors $\zeta_{1}$ and $\zeta_{2}$ do not exceed 1.0 for any wavenumber for any choice of $\theta$ because the central, Galerkin and backward, lumped schemes are unconditionally stable, while the stability conditions of the forward, lumped scheme are satisfied (see Fig.3). 


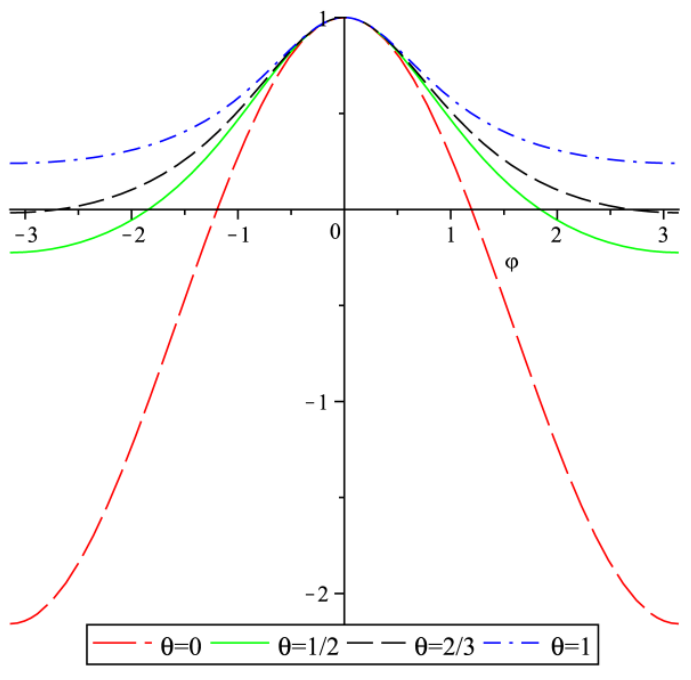

(a)

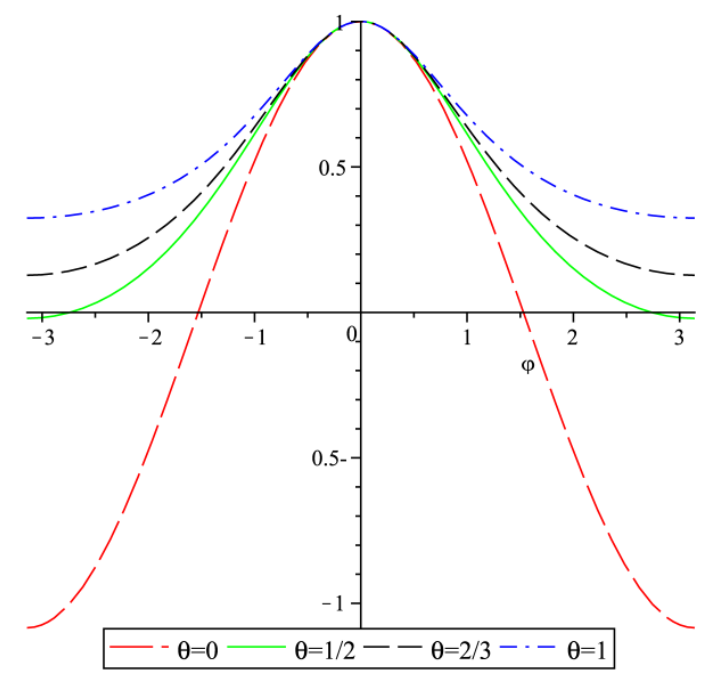

(b)

Fig.4. (a) the amplification factor $\zeta_{1}(\mathrm{~b})$ the amplification factor $\zeta_{2}$ with wavenumber $\varphi \in(-\pi, \pi)$ for the lumped scheme with different choices for $\theta$ when $\Delta x=0.001$ and

$$
\Delta t=500
$$

The amplification factors $\zeta_{1}$ and $\zeta_{2}$ for the forward, lumped scheme increase to exceed 1.0 with the increase of $\Delta t$, i.e. $\zeta_{1}$ exceed 1.0 when $\Delta t>316.6561114$ and $\zeta_{2}$ exceed 1.0 when $\Delta t>479.8464492$, because the stability conditions are not satisfied (see Fig.4).

It should be mentioned that we use Maple software to obtain these plots in Figs.1-4.

\section{Conclusions}

In this work, Fourier stability analysis was performed on two finite element schemes for solving Reaction-diffusion system which describes a reversible chemical reaction. We conclude that the consistent and the lumped finite element schemes are unconditionally stable when $1 / 2 \leq \theta \leq 1$ while the consistent scheme is stable under the conditions $\Delta t \leq(\Delta x)^{2} / 6 a$ and $\Delta t \leq(\Delta x)^{2} / 6 b$ when $\theta=0$. Also, the lumped scheme is stable under the conditions $\Delta t \leq(\Delta x)^{2} / 2 a$ and $\Delta t \leq(\Delta x)^{2} / 2 b$ when $\theta=0$. Therefore, the forward, lumped scheme has better conditional stability properties than the forward, consistent scheme. 


\section{$\underline{\text { REFERENCES }}$}

[1] Anmala, J. and Mohtar, R.H., (2011), Fourier Stability Analysis of TwoDimensional Finite Element Schemes for Shallow Water Equations, Int. J. Comput. Fluid Dyn., 25(2), 75-93.

[2] Dogan, A., (2002), Numerical Solution of RLW Equation Using Linear Finite Elements within Galerkin's Method, Appl. Math. Model., 26, 771-783.

[3] Dogan, A., (2004), A Galerkin Finite Element Approach to Burgers Equation, Appl. Math. Comput., 157, 331-346.

[4] Eymard, R., Hilhorst, D., Murakawa, H., and Olech, M., (2010), Numerical Approximation of a Reaction-Diffusion System with Fast Reversible Reaction, Chin. Ann. Math., 31B(5), 631-654.

[5] Garbey, M., Kaper, H.G. and Romanyukha, N., (2001), A Fast Solver for Systems of Reaction-Diffusion Equations, Thirteenth International Conference on Domain Decomposition Methods, 385-392.

[6] Hirsch, C., (2007), Numerical Computation of Internal and External Flows: Fundamentals of Computational Fluid Dynamics, 2nd ed., ButterworthHeinemann, Amsterdam.

[7] Jaber, F.H. and Mohtar, R.H., (2002), Stability and Accuracy of Finite Element Schemes for the One-Dimensional Kinematic Wave Solution, Adv. Water Res., $25,427-438$.

[8] Lapidus, L., and Pinder, G.F., (1999), Numerical Solution of Partial Differential Equations in Science and Engineering, John Wiley \& Sons, NY.

[9] Segerlind, L.J., (1984), Applied Finite Element Analysis, 2nd ed., John Wiley \& Sons, NY.

[10] Shanthakumar, M., (1989), Computer Based Numerical Analysis, Khanna Publishers, India.

[11] Shi, J., (1993), A Simplified Von Neumann Method for Linear Stability Analysis, College of Aeronautics Report No. 9310, Cranfield Institute of Technology, England. 